\title{
On the relation between modal and multi-modal logics over Lukasiewicz logic
}

\author{
Francesc Esteva and Lluís Godo \\ Artificial Intelligence Research Institute, IIIA \\ Spanish National Research Council, CSIC \\ Bellaterra, Spain \\ Email: \{esteva,godo\}@iiia.csic.es
}

\author{
Ricardo Óscar Rodríguez \\ Computer Science Department \\ University of Buenos Aires \\ Buenos Aires, Argentina \\ Email: ricardo@dc.uba.ar
}

\begin{abstract}
In a previous paper, it was shown that the (minimal) modal logic $\mathrm{ML}_{n}^{c}$ with fuzzy accessibility relations over the finite-valued Lukasiewicz logic $\mathbf{L}_{n}$ and a corresponding multimodal logic $\operatorname{mML}_{n}^{c}$ (with a modality $\square_{a}$ for each value $a$ in the $n$-valued $\mathbf{L}_{n}$-chain) had the same expressive power when the language is extended with truth-constants. In this paper we partially extend these results when replacing the underlying $\operatorname{logic} \mathbf{L}_{n}$ by the infinite-valued Lukasiewicz logic (with rational truth constants in the language). We prove that the (standard) tautologies of the modal logic $\mathrm{ML}^{c}$ (resp. $\mathrm{mML}^{c}$ ) are in fact the common tautologies of all the logics $\mathrm{ML}_{n}^{c}$ (resp. all the logics $\mathrm{mML}_{n}^{c}$ ) when letting $n$ vary over $\mathbb{N}$. This fact opens the door to show an alternative proof of the finite model property for these logics and hence their decidability.
\end{abstract}

\section{Introduction and preliminaries}

Theoretical studies of fuzzy or many-valued modal logics on different issues have attracted an increasing attention in the last years, both following general and foundational approaches e.g. in [16], [2], [3], [7], [14], as well as focusing on particular families of logics, mainly those based on Gödel fuzzy logic [5], [4], [6], [10], [9], on Łukasiewicz logic [13], [1] or even on Product fuzzy logic [17].

In this paper we follow the latter path, and focus our attention on modal logics based on Łukasiewicz logics. In [2] it was shown that the (minimal) modal logic $\mathrm{ME}_{n}^{c}$ with fuzzy accessibility relations over the $n$-valued Łukasiewicz logic $\mathrm{E}_{n}$ and a corresponding multi-modal logic $\mathrm{mME}_{n}^{c}$ (with a modality $\square_{a}$ for each value $a$ in the $n$-valued $\bigsqcup_{n^{-}}$ chain) have the same expressive power when the language is extended with truth-constants. In this paper we partially extend these results when replacing the underlying logic $\mathrm{E}_{n}$ by the infinite-valued Łukasiewicz logic (with rational truth constants in the language). We prove that the (standard) tautologies of the modal logic $\mathrm{ME}^{c}$ (resp. $\mathrm{mME}^{c}$ ) are in fact the common tautologies of all the logics $\mathrm{ME}_{n}^{c}$ (resp. all the logics $\operatorname{mMŁ}_{n}^{c}$ ) when letting $n$ vary over $\mathbb{N}$. From this, it can be shown that these logics enjoy the finite model property and hence they are decidable. Some of the decidability results are in fact new proofs of Hájek's results in [12] about decidability of Fuzzy Description Logics with semantics over the standard MV-chain, and their extensions when rational truth-values are included in the language.

The paper is structured as follows. After this short introduction, we recall in Section 2 the needed and relevant results in [2], [3]. In Section 3, the modal logic $\mathrm{Mt}^{c}$ is introduced and its tautologies are characterized as the intersection of the tautologies of $\mathrm{MŁ}_{n}^{c}$ for every $n \in \mathbb{N}$. In Section 4 a complete calculus is given for its corresponding multi-modal logic. In Section 5 we show some decidability results for these modal systems. We conclude with some remarks and open questions.

\section{Preliminaries}

Let $\mathrm{七}_{n}$ be the $n$-valued Łukasiewicz logic and let $\mathbf{L}_{\mathbf{n}}=$ $\left(L_{n}, \odot, \rightarrow, 0,1\right)$, with $L_{n}=\left\{0, \frac{1}{n-1}, \ldots, \frac{n-2}{n-1}, 1\right\}$, be the $n$ element MV chain. It is well known that $\mathrm{E}_{n}$ is axiomatizable and it is complete with respect to evaluations over $L_{n}$. If we add a truth constant $\bar{a}$ for each $a \in L_{n}$ and suitable bookkeeping axioms, the corresponding logic $\mathrm{E}_{n}^{c}$ is also complete w.r.t. evaluations over $L_{n}$ such that for all $e, e(\bar{a})=a$. In [3] the authors axiomatize the minimal modal logic over $£_{n}^{c}$ with a necessity operator $\square$, denoted by $\mathrm{ML}_{n}^{c}$, by the following axioms and rules:

- The set of axioms is the smallest set closed under substitutions containing

- an axiomatic basis for $\mathrm{E}_{n}$

- book-keeping axioms $\overline{(a * b)} \leftrightarrow \bar{a} * \bar{b}$, for every $a, b \in L_{n}$ and $* \in\{\odot, \rightarrow\}$

- $\square \overline{1}, \square \varphi \wedge \square \psi \rightarrow \square(\varphi \wedge \psi)$

- $\square(\bar{a} \rightarrow \varphi) \rightarrow(\bar{a} \rightarrow \square \varphi)$, for every $a \in L_{n}$

- MP rule, i.e. $\varphi, \varphi \rightarrow \psi \vdash \psi$ and monotonicity rule, i.e. $\vdash \varphi \rightarrow \psi$ implies $\vdash \square \varphi \rightarrow \square \psi$

This logic is complete with respect to the Kripke style semantics defined by structures $\mathscr{M}=\langle W, S,\|\|\rangle$ where:

- $W$ is a non-empty set of possible worlds,

- $S$ is a binary fuzzy relation on $W$ valued in $L_{n}$, and

- \|\|$=\left\{\|\|_{v}\right\}_{v \in W}$, where, for each $v \in W,\|\|_{v}$ is an evaluation function assigning to each propositional variable a value in $L_{n}$. 
As usual the evaluation function is extended to propositional formulas in the usual way in $n$-valued Łukasiewicz logic with truth constants (with $\|\bar{a}\|_{v}=a$ for all $a \in L_{n}$ and $v \in W$ ) and to modal formulas as:

$$
\text { - }\|\square \varphi\|_{w}=\inf \left\{S\left(w, w^{\prime}\right) \rightarrow\|\varphi\|_{w^{\prime}}: w^{\prime} \in W\right\}
$$

A possibility operator $\diamond$ can be defined as usual by duality as $\neg \square \neg$.

On the other hand in [2] a multi-modal system over $\mathrm{七}_{n}^{c}$, that will denoted by $\mathrm{mM}_{n}^{c}$, is defined by adding to $€_{n}^{c}$ a family of necessity operators $\square_{a}$, one for each $a \in$ $L_{n} \backslash\{0\}$. This multi-modal logic have been axiomatized by the following axioms and rules:

- The set of axioms is the smallest set closed under substitutions containing

- an axiomatic basis for $\mathrm{七}_{n}$

- book-keeping axioms: $\overline{(a * b)} \leftrightarrow \bar{a} * \bar{b}$

for every $a, b \in L_{n}$ and $* \in\{\odot, \rightarrow\}$

- $\left(\square_{b} \varphi \wedge \square_{b} \psi\right) \rightarrow \square_{b}(\varphi \wedge \psi)$, $\square_{b}(\bar{a} \rightarrow \varphi) \rightarrow\left(\bar{a} \rightarrow \square_{b} \varphi\right)$, $\square_{b}(\varphi \rightarrow \psi) \rightarrow\left(\square_{b} \varphi \rightarrow \square_{b} \varphi\right)$, for every $a \in L_{n}$ and $b \in L_{n} \backslash\{0\}$

- $\square_{b}(\bar{k} \vee \varphi) \rightarrow\left(\bar{k} \vee \square_{b} \varphi\right)$, with $k$ being the coatom of $L_{n}$, for every $b \in L_{n} \backslash\{0\}$

- $\square_{b_{1}} \varphi \rightarrow \square_{b_{2}} \varphi$, for every $b_{1}, b_{2} \in L_{n} \backslash\{0\}$ such that $b_{1} \leqslant b_{2}$

- MP rule, i.e. $\varphi, \varphi \rightarrow \psi \vdash \psi$ and the necessitation rule: from $\vdash \varphi$ infer $\vdash \square_{b} \varphi$ (for every $b \in L_{n} \backslash\{0\}$ ).

This multi-modal logic was proved to be complete with respect to the same Kripke style semantics above mentioned, only by stipulating the following condition for the $\square_{a}$ modalities: given a structure $\mathscr{M}=\langle W, S,\|\|\rangle$, we define for every $v \in W$ and $a \in L_{n} \backslash\{0\}$,

$$
\text { - }\left\|\square_{a} \varphi\right\|_{v}=\inf \left\{\|\varphi\|_{u}: u \in W, S(v, u) \geqslant a\right\}
$$

Observe that the semantics of each $\square_{a}$ is given by a crisp Kripke model obtained by taking the $a$-cut of $S$ (a crisp relation) as its accessibility relation and thus satisfying axiom $K$, the normality axiom. Moreover we can define the corresponding possibility operators $\nabla_{a}$ as $\neg \square_{a} \neg$ and thus having the following evaluation function:

$$
\text { - }\left\|\diamond_{a} \varphi\right\|_{v}=\sup \left\{\|\varphi\|_{u}: S(v, u) \geqslant a\right\}
$$

As it is costumary, it is assumed above that $\inf \emptyset=1$ and $\sup \emptyset=0$.

Actually, the modal and multi-modal system presented above have the same expressive power. Indeed, as shown in [2], both $\square$ is definable in $\mathrm{mML}_{n}^{c}$ and $\square_{b}$ (for every $b \neq 0$ ) is definable in $\mathrm{MŁ}_{n}^{c}$ :

- $\square \varphi:=\bigwedge\left\{\bar{b} \rightarrow \square_{b} \varphi: b \in L_{n} \backslash\{0\}\right\}$,

- $\square_{b} \varphi:=\bigwedge\left\{\left(\bar{b} \rightarrow \diamond(\varphi \leftrightarrow \bar{b})^{n-1}\right)^{n-1} \rightarrow \bar{b}: b \in L_{n}\right\}$.

\section{About the modal logic $M \mathbf{L}^{c}$ with standard semantics}

In this section we consider the minimal modal logic $\mathrm{MŁ}^{c}$ and its relation to the finite-valued minimal modal logics $\mathrm{MŁ}_{n}^{c}$ defined in the previous section. Note that the language of $\mathrm{ML}^{c}$ is the one of Łukasiewicz propositional logic expanded with rational truth constants (a truth constant $\bar{a}$ for every $a \in \mathbb{Q} \cap[0,1]$ ) and a modal operator $\square$. The semantics of $\mathrm{MŁ}^{c}$ is also defined by Kripke models $\mathscr{M}=\langle W, S,\|\|\rangle$ where now $S: W \times W \rightarrow[0,1]$ is a $[0,1]$-valued accessibility relation and, for each $w \in W,\|\|_{w}$ is an evaluation function assigning to each propositional variable a value in $[0,1]$. As in the previous cases, \|\|$_{v}$ is extended to formulas in the usual way, taking into account that $\|\bar{a}\|_{v}=a$ for all rational $a \in[0,1]$ and

$$
\text { - }\|\square \varphi\|_{w}=\inf \left\{S\left(w, w^{\prime}\right) \rightarrow\|\varphi\|_{w^{\prime}}: w^{\prime} \in W\right\}
$$

A possibility operator $\diamond$ is still defined as $\neg \square \neg$.

So far, a strongly complete axiomatization of $\mathrm{MŁ}^{c}$ is not known. However, the rest of this section is devoted to prove the next theorem that, similarly to what happens with the propositional logics, shows that the tautologies of $\mathrm{ML}^{c}$ are actually the common tautologies of (countably many) finite-valued logics $\mathrm{MŁ}_{n}^{c}$.

Theorem 1. A modal formula $\varphi$ is a $\mathrm{ME}^{c}$-tautology iff it is an $\mathrm{M} €_{n}^{c}$-tautology for all natural $n \geqslant 2$ such that $\{a \in \mathbb{Q} \cap$ $[0,1]: \bar{a}$ appears in $\varphi\} \subseteq L_{n}$. In particular, the tautologies of $\mathrm{M} E$ (i.e. without truth-constants) are exactly the common tautologies of all the $\mathrm{M}_{n}$ 's.

Note that the languages of $\mathrm{MŁ}^{c}$ and $\mathrm{ME}_{n}^{c}$ are not the same due to the presence of different truth-constants. This is the reason of the constraint introduced in the above theorem on the $n$ 's.

The fact that any $\mathrm{MŁ}^{c}$-tautology $\varphi$ is a $\mathrm{MŁ}_{n}^{c}$-tautology (provided that all constants in $\varphi$ belong to $L_{n}$ ) is an easy consequence of the following facts:

- the algebra $[0,1]_{\mathrm{E}}$ restricted to $L_{n}=$ $\left\{0, \frac{1}{n-1}, \ldots, \frac{n-2}{n-1}, 1\right\}$ is an $\mathrm{E}_{n}$-chain;

- each fuzzy relation $S: W \times W \longrightarrow L_{n}$ can be considered in fact as a $[0,1]$-valued fuzzy relation as well;

- hence, any $L_{n}$-valued Kripke model can be indeed considered a $[0,1]$-valued model as well.

In order to prove the converse inclusion we will use results of Hájek in [11, pp. 135-137] proving that the first order $[0,1]_{€}$-tautologies coincide with the common first order $€_{n^{-}}$ tautologies for all $n \geqslant 2$. First we need an auxiliary result.

Lemma 1. Let $n$ be any odd natural number greater than 2 and let $f_{n}$ be the function $f_{n}:[0,1] \longrightarrow L_{n}$ defined by:

$$
f_{n}(x)= \begin{cases}0, & \text { if } x=0, \\ \frac{k}{n-1}, & \text { if } x \in\left(\frac{k-1}{n-1}, \frac{k}{n-1}\right] \text { and } 1 \leqslant k \leqslant \frac{n-1}{2} \\ \frac{k}{n-1}, & \text { if } x \in\left[\frac{k}{n-1}, \frac{k+1}{n-1}\right) \text { and } n-1 \geqslant k \geqslant \frac{n-1}{2} \\ 1, & \text { if } x=1,\end{cases}
$$


where $k$ ranges over natural numbers. Then for any $a, b, c \in$ $[0,1]$, if $a \odot b \leqslant c$ then $f_{n}(a) \odot f_{n}(b) \leqslant f_{n}(c)$.

The lemma is proved by an easy computation by cases. As a corollary we obtain the following corollary.

Corollary 1. For each odd natural number $n>2$, if $S$ is a [0,1]-valued fuzzy relation on a set $W$, the relation $S_{n}$ defined by $S_{n}(u, v)=f_{n}(S(u, v))$, is a $L_{n}$-valued fuzzy relation on $W$. Moreover if $S$ further satisfies reflexivity, symmetry, $\odot$-transitivity, or separability, then $S_{n}$ satisfies the same properties.

Recall that $S$ is reflexive if $S(u, u)=1$ for all $u \in W$; it is symmetric if $S(u, v)=S(v, u)$ for all $u, v \in W$; it is $\odot-$ transitive if $S(u, v) \odot S(v, w) \leqslant S(u, w)$ for all $u, v, w \in W$; and it is separable, if $S(u, v)=1$ iff $u=v$.

Now we adapt the notion of distance between first order models in [11, Def. 5.4.28]) to define a distance between Kripke models (where the fuzzy accessibility relation is the only binary predicate). Let $\mathscr{M}=\langle W, S,\|\|\rangle$ and $\mathscr{M}^{\prime}=\left\langle W, S^{\prime},\|\|^{\prime}\right\rangle$ be two $[0,1]$-valued Kripke models with the same set of worlds. We define

(i) $d\left(S, S^{\prime}\right)=\sup _{v, v^{\prime} \in W}\left\{\left|S\left(v, v^{\prime}\right)-S^{\prime}\left(v, v^{\prime}\right)\right|\right\}$,

(ii) $d(p)=\sum_{v \in W}\left|\|p\|_{v}-\|p\|_{v}^{\prime}\right|$, for each variable $p$. Finally, for a finite set of variables $X \subset V a r$, define:

$$
d_{X}\left(\mathscr{M}, \mathscr{M}^{\prime}\right)=d\left(S, S^{\prime}\right)+\sum_{p \in X} d(p) .
$$

Note that this distance can be applied indistinctly if $M$ and/or $M^{\prime}$ are $L_{n}$-valued models.

Lemma 2. (i) Let $X$ be a finite set of variables. For each $\delta>0$ and each $[0,1]$-valued Kripke model $\mathscr{M}=\langle W, S,\|\|\rangle$, there exists an odd natural number $n>2$ and a $L_{n}$-valued Kripke model $\mathscr{M}_{n}=\left\langle W, S_{n},\|\|^{n}\right\rangle$ such that $d_{X}\left(\mathscr{M}, \mathscr{M}_{n}\right)<\delta$, where $S_{n}=f_{n} \circ S$ and $\|p\|_{v}^{n}=f_{n}\left(\|p\|_{v}\right)$ for any world $v$ and propositional variable $p$.

(ii) For each formula $\varphi,[0,1]$-valued Kripke model $\mathscr{M}=$ $\langle W, S,\|\|\rangle$ and $\varepsilon>0$, there is $n>2$ for which the $L_{n}$-valued Kripke model $\mathscr{M}_{n}=\left\langle W, S_{n},\|\|^{n}\right\rangle$ satisfies

$$
\left|\|\varphi\|_{v}-\|\varphi\|_{v}^{n}\right|<\varepsilon
$$

for each world $v \in W$.

Proof. (i) Let $k$ be the cardinality of $X$. Then, by construction, $d_{X}\left(\mathscr{M}, \mathscr{M}_{n}\right)=d\left(S, S_{n}\right)+\sum_{p \in X} d(p) \leqslant \frac{1}{n-1}+\frac{k}{n-1}=$ $\frac{k+1}{n-1}$. Then it is enough to take $n>\frac{k+1}{\delta}+1$.

(ii) Let $X$ be the set of variables appearing in $\varphi$. Define the complexity of $\varphi$ as follows: $\tau(\varphi)=0$ if $\varphi$ is propositional variable or a constant, $\tau(\varphi \rightarrow \psi)=$ $\max (\tau(\varphi), \tau(\psi))+1$ and $\tau(\square \varphi)=\tau(\varphi)$. Now, for a fixed $\varepsilon>0$, take $\delta=\frac{\varepsilon}{2^{\tau(\varphi)}}$. Then, if we apply [11, Lemma 5.4.29], using $d_{X}\left(\mathscr{M}, \mathscr{M}_{n}\right)$, we obtain for each world $v \in W$ that the following inequality holds: $\left|\|\varphi\|_{v}-\|\varphi\|_{v}^{n}\right|<\varepsilon$.

And now we are ready to prove the theorem. We have already proved one inclusion. To prove the other one, suppose that $\varphi$, in the language of $\mathrm{MŁ}^{c}$, is not an $\mathrm{MŁ}^{c}$-tautology, i.e., there is a Kripke model $\mathscr{M}$ and a world $w \in W$ such that $\|\varphi\|_{w}<1$. By Lemma 2, taking $\varepsilon<1-\|\varphi\|_{w}$, there is an odd natural $n$ and a $L_{n}$-valued model $\mathscr{M}_{n}$ such that $\|\varphi\|_{w}^{n}<1$. This completes the proof of Theorem 1 .

As an easy corollary we have the following.

Corollary 2. Theorem 1 is also valid if the Kripke models are restricted to models such that the accessibility relations satisfy either reflexivity, symmetry, transitivity or separability, or they are restricted to similarity relations.

We finish the section with two open problems. Due to the completeness result for $\mathrm{MŁ}_{n}^{c}$, it is easy to prove that $\mathrm{MŁ}_{n}^{c}$ is in fact a conservative expansion of $€_{n}$. Then we have a first open problem:

(P1) Is $\mathrm{MŁ}^{c}$ is a conservative expansion of $€$ ?

Now, let $\mathscr{L}$ be the axiomatic system defined taking as axioms the smallest set closed under substitutions containing;

- $\quad$ an axiomatic basis for $€$

- $\quad$ book-keeping axioms $(\bar{a} * \bar{b}) \leftrightarrow(\overline{a * b})$, for all rationals $a, b \in[0,1]$ and $* \in\{\odot, \rightarrow\}$,

- $\square \overline{1},(\square \varphi \wedge \square \psi) \rightarrow \square(\varphi \wedge \psi)$

- $\square(\bar{a} \rightarrow \varphi) \leftrightarrow(\bar{a} \rightarrow \square \varphi)$, for every rational $a \in[0,1]$

and having as rules MP and the monotonicity rule: from $\vdash \varphi \rightarrow \psi$ derive $\vdash \square \varphi \rightarrow \square \psi$. The second open question is:

(P2) Is $\mathscr{L}$ an axiomatization (at least for theorems) of $\mathrm{ME}^{c}$ ?

\section{The multi-modal approach}

In the introduction we have recalled the finite-valued multimodal logic $\mathrm{mM七}_{n}^{c}$, where we have for each $a \in L_{n}$ both a truth constant $\bar{a}$ and a modal operator $\square_{a}$. We have also mentioned that $\mathrm{mMY}_{n}^{c}$ is complete with respect to a corresponding class of $L_{n}$-valued Kripke models, and that it has the same expressive power as $\mathrm{ML}_{n}^{c}$.

In this section we first define an expansion of $\mathrm{mMŁ}_{n}^{c}$ by introducing in the language a modal operator $\square_{a}$ for each rational $a \in[0,1]$. The semantics for this system, that we will denote by $\mathrm{mMŁ}_{n}^{+}$, is as for $\mathrm{mMŁ}_{n}^{c}$ with one exception: Kripke models for $\mathrm{mML}_{n}^{+}$will be structures of the form $\mathscr{M}=\langle W, S,\|\|\rangle$, where $W$ and \|\| are as in $\mathrm{mML}_{n}^{c}$ structures, but now the accessibility relation $S$ is $[0,1]$-valued. Thus for any rational $a \in[0,1]$, the truthevaluation of a formula $\square_{a} \varphi$ in a world $v \in W$ will be $\left\|\square_{a} \varphi\right\|_{v}=\inf \left\{\|\varphi\|_{u}: u \in W, S(v, u) \geqslant a\right\} \in L_{n}$.

Taking inspiration in the axiomatizations in [8] and in [3] for modal logics with crisp accessibility relations, we introduce the following axiomatic system for the multimodal $\operatorname{logic} \mathrm{mME}_{n}^{+}$.

Definition 1. The set of axioms of $\mathrm{mM}_{n}^{+}$is the smallest set closed under substitutions containing:

- axioms of $\epsilon_{n}$ with truth constants,

- $\square_{a}(\varphi \wedge \psi) \rightarrow\left(\square_{a} \varphi \wedge \square_{a} \psi\right)$,

- $\square_{a}(\bar{k} \rightarrow \psi) \leftrightarrow\left(\bar{k} \rightarrow \square_{a} \psi\right)$, for each $k \in L_{n}$. 
- $\square_{a}(\bar{k} \vee \psi) \rightarrow\left(\bar{k} \vee \square_{a} \psi\right)$, where $k=\frac{n-2}{n-1}$ is the coatom of $L_{n}$,

- $\square_{a} \varphi \rightarrow \square_{b} \varphi$, for all $b \geqslant a$

Rules of $\mathrm{mML}_{n}^{+}$are MP, the monotonicity rule: $\vdash \varphi \rightarrow \psi$ implies $\vdash \square_{a} \varphi \rightarrow \square_{a} \psi$, for any rational $a \in[0,1]$, and the continuity (infinitary) rule: $\left\{\diamond_{b} \varphi: \forall b<a\right\} \vdash \diamond_{a} \varphi$.

It is easy to check that the axioms and inference rules of $\mathrm{mML}_{n}^{+}$are sound with respect to the semantic just defined. It is worth noticing the logic $\mathrm{mMI}_{n}^{+}$is a bit redundant for interpreting a set of infinite dense modalities with a finite number of truth values. To prove completeness we use the canonical model technique and to this end we define the canonical model by the following steps:

1) Define $W_{c a n}$ as the set of propositional homomorphisms of modal formulas into $L_{n}$ (taking as new propositional variables all the formulas starting with $\square)$ evaluating all theorems of $\mathrm{mME}_{n}^{+}$to 1 . Notice that for these homomorphisms we can easily verify that for all $v_{1}, v_{2} \in W_{c a n}, v_{1}=v_{2}$ iff $\left\{\varphi: v_{1}(\varphi)=\right.$ $1\}=\left\{\varphi: v_{2}(\varphi)=1\right\}$.

2) For each rational $a \in[0,1]$, define $S_{c a n}^{a} \subseteq W_{c a n} \times W_{c a n}$ by $\left(v_{1}, v_{2}\right) \in S_{\text {can }}^{a}$ iff for all $\varphi, v_{1}\left(\square_{a} \varphi\right) \leqslant v_{2}(\varphi)$, or equivalently, iff $v_{1}\left(\square_{a} \varphi\right)=1$ implies $v_{2}(\varphi)=1$, cf. [3, Prop. 4.1]. The relations $S_{c a n}^{a}$ verify the following properties:

$$
\begin{aligned}
& \text { - } \quad \text { If } b \leqslant a \text { then } S_{c a n}^{a} \subseteq S_{c a n}^{b}, \\
& \text { - } \quad S_{c a n}^{0}=W_{c a n} \times W_{c a n},
\end{aligned}
$$

3) Define the fuzzy accessibility relation of the canonical model $S_{\text {can }}$ by putting, for every $v_{1}, v_{2} \in W_{c a n}$ :

$$
S_{c a n}\left(v_{1}, v_{2}\right)=\sup \left\{a:\left(v_{1}, v_{2}\right) \in S_{c a n}^{a}\right\}
$$

Lemma 3. The fuzzy relation $S_{\text {can }}$ satisfies the following property:

- for each $a \in L_{n}, S_{\text {can }}^{a}$ is the a-cut of the relation $S_{c a n}$.

Proof. To prove this lemma we need the continuity rule. It is well known that, for each rational $a, S_{c a n}^{a}$ is the $a$-cut of the relation $S_{c a n}$ if and only if, $\cap\left\{S_{c a n}^{b}: b<a\right\}=S_{c a n}^{a}$. Obviously $S_{\text {can }}^{a} \subseteq \cap\left\{S_{\text {can }}^{b}: b<a\right\}$. Suppose then that $\cap\left\{S_{\text {can }}^{b}: b<a\right\} \subset$ $S_{c a n}^{a}$. In such a case there is an $\left(u_{1}, u_{2}\right) \in \cap\left\{S_{\text {can }}^{b}: b<a\right\}$ that does not belong to $S_{\text {can }}^{a}$. Take a formula $\varphi$ such that $u_{2}(\varphi)=1$ and $u(\varphi) \neq 1$ for all $u \neq u_{2}$. Such a formula always exists by McNaughton theorem. It is obvious that $\nabla_{b} \varphi$ is a theorem for all $b<a$ but $\nabla_{a} \varphi$ is not a theorem, in contradiction with the continuity rule.

The canonical model is then defined as the triple $\left\langle W_{c a n}, S_{c a n},\|\|{ }^{c a n}\right\rangle$, where for every $v \in W_{c a n},\|p\|_{v}^{c a n}=v(p)$ for each propositional variable $p$ and $\|\bar{a}\|_{v}^{c a n}=a$ for every rational $a \in[0,1]$.

Lemma 4. (Truth lemma) For any formula $\varphi$ in the multimodal language and any $v \in W_{\text {can }},\|\varphi\|_{v}^{c a n}=v(\varphi)$.

Proof. The proof follows the same steps as in [2, Lemma 3.5].
As a consequence we have the following completeness result.

Theorem 2. For any $n, \mathrm{mML}_{n}^{+}$is complete for theorems with respect to the intended $L_{n}$-valued Kripke semantics.

Due to this completeness result the following two statements hold true as well:

- If we consider the fragment of $\mathrm{mMt}_{n}^{c}$ with only one necessity operator $\square_{a}$, then it would be equivalent to the fragment with only another operator $\square_{b}$, since they would be both complete with respect to the same class of crisp Kripke models.

- The logic $\mathrm{mMt}_{n}^{+}$is a conservative expansion of both $\mathrm{mME}_{n}^{c}$ and all the previous fragments with only one necessity operator $\square_{a}$.

Moreover we could consider the logic $\mathrm{mMŁ}^{+}$defined as $\mathrm{mM七}_{n}^{+}$but replacing $\mathrm{Ł}_{n}$ as base logic by $€$. Although we do not have an axiomatization of this logic, the semantics is clear and defined accordingly, and thus we can consider the set of its tautologies. Then we have the analogous of Theorem 1 for the multimodal setting.

Theorem 3. A multi-modal formula $\varphi$ is a tautology of $\mathrm{mML}^{+}$iff it is a tautology of $\mathrm{mM}_{n}^{+}$for all natural $n \geqslant 2$ such that $\{a \in \mathbb{Q} \cap[0,1]: \bar{a}$ appears in $\varphi\} \subseteq L_{n}$.

The proof of this theorem is easier since in this case the Kripke frames for both logics are the same (in both cases the accessibility relations are $[0,1]$-valued relations), and thus Hájek's result directly applies.

Finally, we notice that the $\square$ operator in the modal logic $\mathrm{ME}^{c}$ can be semantically captured in the multimodal logic $\mathrm{mME}^{c}$ in the following (infinitary) sense.

Proposition 1. Given a $[0,1]$-valued Kripke model $M=$ $(W, S,\|\|)$, the following holds for every world $v \in W$ :

$$
\|\square \varphi\|_{v}=\inf \left\{\left\|\bar{a} \rightarrow \square_{a} \varphi\right\|_{v}: a \in \mathbb{Q} \cap[0,1]\right\}
$$

Proof. Indeed, we have:

$\inf \left\{\left\|\bar{a} \rightarrow \square_{a} \varphi\right\|_{v}: a \in \mathbb{Q} \cap[0,1]\right\}=$

$=\inf _{a}\left(a \rightarrow \inf _{v^{\prime}}\left\{S_{a}\left(v, v^{\prime}\right) \rightarrow\|\varphi\|_{v^{\prime}}\right\}\right)=$

$=\inf _{a}\left(\inf _{v^{\prime}}\left\{\left(a \rightarrow\left(S_{a}\left(v, v^{\prime}\right) \rightarrow\|\varphi\|_{v^{\prime}}\right)\right\}\right)=\right.$

$=\inf _{a}\left(\inf _{v^{\prime}}\left\{S_{a}\left(v, v^{\prime}\right) \rightarrow\left(a \rightarrow\|\varphi\|_{v^{\prime}}\right)\right\}\right)=$

$=\inf _{v^{\prime}}\left\{\inf _{a}\left(a \rightarrow\|\varphi\|_{v^{\prime}}\right): S\left(v, v^{\prime}\right) \geqslant a\right\}=$

$=\inf _{v^{\prime}}\left\{\sup \left\{a: S\left(v, v^{\prime}\right) \geqslant a\right\} \rightarrow\|\varphi\|_{v^{\prime}}\right\}=$

$=\inf _{v^{\prime}}\left\{S\left(v, v^{\prime}\right) \rightarrow\|\varphi\|_{v^{\prime}}\right\}=\|\square \varphi\|_{v}$.

Thus, in $\mathrm{mME}^{c}$, one could say that $\square \varphi$ is semantically definable as the infinitary conjunction $\bigwedge\left\{\bar{a} \rightarrow \square_{a} \varphi: a \in \mathbb{Q} \cap\right.$ $[0,1])\}$.

\section{Decidability of Mt}

In this section, when talking about a modal logic we will actually refer to its set of valid formulas. In what follows, we prove that the minimal modal logic MŁ and some of its conservative extensions have the finite model property (FMP in short). This result was first reported by 
Hájek in [12], but there, he uses a systematic reduction of problems of satisfiability and validity in Description logic to the same problems in Propositional logic. Instead of that reduction, we propose to use a many-valued generalization of the classical filtration method. We understand that this generalization is itself a technical contribution which could be useful for future research.

Coming back to the main goal in this section, recall that we say a logic $L$ has the FMP if for every formula $\varphi$ that does not belong to $L$ (i.e. it is not $L$-valid), there exists a finite $L$-model $\mathscr{M}$ where $\varphi$ is not valid in $\mathscr{M}$. To prove that MŁ has the FMP, we proceed in the following way. First, we assume that a formula $\varphi$ is not valid in the class of all $[0,1]_{\mathrm{E}}$-valued Kripke models, hence, there exists a $[0,1]_{\mathrm{七}^{-}}$ valued model $\mathscr{M}=\langle W, S,\|\|\rangle$ and a world $v \in W$, such that $\|\varphi\|_{v}<1$. According to Lemma 2, there is an odd natural $n$ and a $L_{n}$-valued model $\mathscr{M}_{n}$ such that $\|\varphi\|_{v}<1$. To complete the proof, we apply a filtration on the $L_{n}$-valued model $\mathscr{M}_{n}$ to obtain a finite model $\mathscr{M}_{n}^{\star}$ such that $\|\varphi\|_{[v]}^{\star}<1$.

To this end, we need to introduce the notion of filtration. We start by defining, for any formula $\varphi$, the set $\Phi_{\varphi}$ of all sub-formulas of $\varphi$. Note that $\Phi_{\varphi}$ is always finite and it also has the property of being closed under sub-formulae. We have also to introduce the notion of equivalent worlds and the classes of equivalence of worlds, in a model:

Given a model $\mathscr{M}=\langle W, S,\|\|\rangle$ and a set of formulae $\Phi$ we say that $w$ and $w^{\prime}$ are equivalent worlds with respect to $\mathscr{M}$ and $\Phi$ (written $w \cong_{\Phi} w^{\prime}$ ) if and only if for all formulae $\psi \in \Phi:\|\psi\|_{w}=\|\psi\|_{w^{\prime}}$ (note that we don't say anything about the formulae that are not in $\Phi)$. We shall say that a subset of $W$ is the $\Phi$-equivalence class of $w$ (in $W$ ) if and only if it consists of all and only those worlds in $W$ which are equivalent to $w$ with respect to $\Phi$. We use the notation $[w]$ for this equivalence class. Analogously, we can define $[\psi]$ for a formula $\psi$ as the set of all worlds in $W$ which are $\psi$-equivalent.

Next step is to define a filtration with respect to a set of formulae $\Phi$ which is closed under sub-formulae.

Definition 2. The $\Phi$-filtration of a (fuzzy) Kripke model $\mathscr{M}$ $=\langle W, S,\|\|\rangle$ through $\Phi$ is another (fuzzy) Kripke model $\mathscr{M}^{\star}$ $=\left\langle W^{\star}, S^{\star},\|\|^{\star}\right\rangle$ such that:

1) $W^{\star}=\{[w] \mid w \in W\}$, where $[w]$ denotes the equivalence class of $w$ wrt $\Phi$.

2) \|\|$^{\star}$ is defined as the original \|\| , restricted to the atomic formulas in $\Phi$ and the worlds in $W^{\star}$. Thus, $\|p\|_{[w]}^{\star}=\|p\|_{w}$, if $p$ is a propositional variable in $\Phi$ and $[w] \in W^{\star}$.

3) $S\left(w, w^{\prime}\right) \leqslant S^{\star}\left([w],\left[w^{\prime}\right]\right)$, for all $w, w^{\prime} \in W$.

4) $S^{\star}\left([w],\left[w^{\prime}\right]\right) \odot\|\square \psi\|_{w} \leqslant\|\psi\|_{w^{\prime}}$, for all $w, w^{\prime} \in W$.

We can now state and prove the fundamental theorem related to the filtration.

Theorem 4. Suppose $\mathscr{M}=\langle W, S,\|\|\rangle$ is any model, $\Phi$ is any set of formulae (not necessarily finite) which is closed under sub-formulae, and $\mathscr{M}^{\star}=\left\langle W^{\star}, S^{\star},\|\|^{\star}\right\rangle$ is any filtration of $\mathscr{M}$ through $\Phi$. Then for every formula $\psi \in \Phi$ and for every $w \in W$,

$$
\|\psi\|_{[w]}^{\star}=\|\psi\|_{w}
$$

Proof. The proof is by structural induction over modal formulas $\psi \in \Phi$. If $\psi$ is a propositional variable, the theorem holds by the definition of \|\|$^{\star}$ in a filtration. The relevant induction step is when $\psi=\square \theta$. We divide the proof in two cases:

$\leqslant)$ By definition,

$$
\begin{gathered}
\|\square \theta\|_{[w]}^{\star}=\inf _{\left[w^{\prime}\right]}\left\{S^{\star}\left([w],\left[w^{\prime}\right]\right) \rightarrow\|\theta\|_{\left[w^{\prime}\right]}^{\star}\right\} \stackrel{I H}{=} \\
\inf _{\left[w^{\prime}\right]}\left\{S^{\star}\left([w],\left[w^{\prime}\right]\right) \rightarrow\|\theta\|_{w^{\prime}}\right\} .
\end{gathered}
$$

By condition 3 in Definition 2 the antecedent of the last implication is greater than $S\left(w, w^{\prime}\right)$. Then, replacing $S^{\star}$ with $S$, we obtain that the last expresion is less or equal to:

$$
\inf _{w^{\prime}}\left\{S\left(w, w^{\prime}\right) \rightarrow\|\theta\|_{w^{\prime}}\right\}=\|\square \theta\|_{w} .
$$

$\geqslant \quad$ By condition 4 in Definition 2, we know that for all $w^{\prime} \in W:$

$$
\|\square \theta\|_{w} \leqslant S^{\star}\left([w],\left[w^{\prime}\right]\right) \rightarrow\|\theta\|_{w^{\prime}} \stackrel{I H}{=} S^{\star}\left([w],\left[w^{\prime}\right]\right) \rightarrow\|\theta\|_{\left[w^{\prime}\right]}^{\star} .
$$

Hence the left side of the last inequation is independent of $\left[w^{\prime}\right]$, then we are able to take infimum on the right side. Thus, we obtain:

$$
\|\square \theta\|_{w} \leqslant \inf _{\left[w^{\prime}\right]}\left\{S^{\star}\left([w],\left[w^{\prime}\right]\right) \rightarrow\|\theta\|_{\left[w^{\prime}\right]}^{\star}\right\}=\|\square \theta\|_{[w]}^{\star} .
$$

In general, conditions 3 and 4 in Definition 2 do not uniquely determine $S^{\star}$. Actually, they allow us to choose any fuzzy relation $S^{\star}$ in the interval $\underline{S} \leqslant S^{\star} \leqslant \bar{S}$ where:

$$
\begin{gathered}
\underline{S}([x],[y])=\sup _{v \in[x]} \sup _{u \in[y]}\{S(v, u)\} \\
\bar{S}([x],[y])=\inf _{\square \theta \in \Phi}\left\{\|\square \theta\|_{x} \rightarrow\|\theta\|_{y}\right\} .
\end{gathered}
$$

Indeed, $\underline{S}$ and $\bar{S}$ satisfy conditions 3 and 4 in Definition 2 , respectively. The fact that $\underline{S}$ satisfies condition 4 can be shown in the following way. By definition of satisfiability in a world, we have:

$$
\forall x, y \in W:\|\square \theta\|_{x} \odot S(x, y) \leqslant\|\theta\|_{y} .
$$

In addition, by definition of classes of equivalence and because of $\square \theta \in \Phi$, we know that $\forall v \in[x]:\|\square \theta\|_{v}=\|\square \theta\|_{x}$ and $\forall u \in[y]:\|\theta\|_{u}=\|\theta\|_{y}$. Then, we can rewrite the previous inequation as follows:

$$
\forall v \in[x] \text { and } \forall u \in[y]:\|\square \theta\|_{v} \odot S(x, y) \leqslant\|\theta\|_{u} .
$$

In particular, we are able to point out that:

$$
\|\square \theta\|_{x} \odot \sup _{v \in[x]} \sup _{u \in[y]} S(v, u) \leqslant\|\theta\|_{y}
$$


which agrees with condition 4 for $\underline{S}$.

In order to prove that $\bar{S}$ satisfies condition 3, we can proceed in the following way. Again by definition of satisfiability in a world, we have:

$$
\begin{gathered}
\forall x, y \in W: S(x, y) \leqslant \inf _{\square \theta \in \mathscr{L}}\left\{\|\square \theta\|_{x} \rightarrow\|\theta\|_{y}\right\} \leqslant \\
\inf _{\square \theta \in \Phi}\left\{\|\square \theta\|_{x} \rightarrow\|\theta\|_{y}\right\}=\bar{S}([x],[y]) .
\end{gathered}
$$

As in the classical case, the filtration defined by $\underline{S}$ will be called the coarsest and the filtration defined by $\bar{S}$ will be called the greatest.

It is worth mentioning that a relation $S^{\star}$ between $\underline{S}$ and $\bar{S}$ may be neither symmetrical nor transitive even if the original $S$ is. However, we are always able to construct such relations from filtrations as the following proposition proves.

Proposition 2. Given a symmetrical (resp. transitive) fuzzy Kripke model we can always define a symmetrical (resp. transitive) filtration of the original model.

Proof. It is enough to consider the coarsest filtration with $\underline{S}$ which is symmetrical (resp. transitive) if the original relation $S$ is symmetrical (resp. transitive).

A very important property of filtrations is that they are finite whenever the "filter" $\Phi$ is finite as it is shown in the next proposition.

Proposition 3. Suppose $\mathscr{M}^{\star}$ is a filtration of a $L_{n}$-valued Kripke model $\mathscr{M}$ through a set $\Phi$ which is finite. Then $\mathscr{M}^{\star}$ contains at most $n^{|\Phi|}$ worlds.

As an immediate consequence of Proposition 3, we have the following result.

Theorem 5. The logic MŁ is decidable.

Combining Propositions 2 and 3, we are able to prove that the logics of several subclasses of fuzzy Kripke models have the FMP. These results do not immediately follow using Hájek's approach because it is not clear what happens when new axioms involving roles are included. Moreover, we can extend the last result for $\mathrm{ME}^{c}$ by including the expression $\forall a \in L_{n}:\|\bar{a}\|_{[w]}=a$ in Condition 2 of Definition 2. In addition, since the logic $\mathrm{mMK}_{n}^{c}$ shares the same semantics than the $\operatorname{logic} \mathrm{MŁ}^{c}$, we are also able to use the previous ideas in order to prove that $\mathrm{mML}_{n}^{c}$ is also decidable. But in that case, we have to replace Condition 4 in Definition 2 with the following one: for any $\alpha \in \mathbb{Q} \cap[0,1]$,

$\left.4^{\prime}\right) \quad$ If $S^{\star}\left([w],\left[w^{\prime}\right]\right)=\alpha$ then $\|\psi\|_{w^{\prime}} \geqslant\left\|\square_{\alpha} \psi\right\|_{w}$.

Corollary 3. $\mathrm{M}^{c}$ and $\mathrm{mM}_{n}^{c}$ are decidable.

\section{Conclusions and future work}

In this paper we have been basically concerned with minimal modal and multimodal logics defined over the infinitely valued Łukasiewicz logic $€$ with truth constants, $\mathrm{ME}^{c}$ and $\mathrm{mME}^{c}$ respectively. We have shown that the tautologies of these modal logics can be obtained as the common tautologies of corresponding modal logics over finite-valued Łukasiewicz logics $\biguplus_{n}$ for countably many $n$ 's, thus generalising the well-known result for the propositional case. Moreover, using a filtration method this result allows us to show the FMP, and hence decidability as well, for these logics. These results are not actually novel, they follow from Hájek's results on Fuzzy Description logics [12], but we think the alternative proof we offer may be of more general application when considering classes of Kripke models with accessibility relations satisfying further properties. Also, in the paper we have pointed out to several open problems, among them to find an axiomatization for $\mathrm{ML}^{c}$. These will be topics of future research.

Acknowledgments. The authors are grateful to two anonymous reviewers for their helpful and detailed reviews. They acknowledge partial support by the H2020-MSCARISE-2015 project SYSMICS, the Spanish MINECO project RASO (TIN2015-71799-C2-1-P) and the Argentinean project PIP CONICET 11220150100412CO.

\section{References}

[1] F. Bou, M. Cerami and F. Esteva. Finite-valued Łukasiewicz modal logic is PSPACE-complete. Proc. of IJCAI'11, Vol. 2, 774-779, 2011.

[2] F. Bou, F. Esteva, L. Godo, R. Rodríguez. Characterizing Fuzzy Modal Semantics through a Crisp Multimodal System. Proc. of IFSAEUSFLAT 2009, 1541-1546.

[3] F. Bou, F. Esteva, L. Godo, R. Rodríguez. On the Minimum ManyValued Modal Logic over a Finite Residuated Lattice. Journal of Logic and Computation 5:21, 739-790. 2011

[4] X. Caicedo, G. Metcalfe, R.O. Rodríguez, J. Rogger. A finite model property for Gödel modal logics. Proc. of TACL 2013, 183-186, 2013.

[5] X. Caicedo, R. O. Rodríguez. Standard Gödel Modal Logics. Studia Logica 94(2): 189-214 (2010)

[6] X. Caicedo, R.O. Rodríguez. Bi-modal Gödel logic over [0, 1]-valued Kripke frames. J. Log. Comput. 25(1): 37-55 (2015)

[7] P. Cintula, C. Noguera, J. Rogger. From Kripke to Neighborhood Semantics for Modal Fuzzy Logics. Proc of IPMU'16, Vol. 2, 95107, 2016.

[8] F. Esteva, P. Garcia, L. Godo, R. Rodríguez. A modal account of similarity-based reasoning. International Journal of Approximate Reasoning 16 (3-4), 235-260, 1997.

[9] T.-F. Fan. Fuzzy Bisimulation for Gödel Modal Logic. IEEE Trans. Fuzzy Systems 23(6): 2387-2396 (2015)

[10] T.-F. Fan, C.J. Liau. Logical characterizations of regular equivalence in weighted social networks. Artif. Intell. 214: 66-88 (2014)

[11] P. Hájek. Metamathematics of Fuzzy Logic. Trends in Logic 4, Kluwer, 1998.

[12] P. Hájek. Making fuzzy description logic more general. Fuzzy Sets and Systems 154, 1-15. 2005.

[13] G. Hansoul and B. Teheux. Extending Lukasiewicz logics with a modality: Algebraic approach to relational semantics. Studia Logica, 101(3): 505-545, 2013.

[14] M. Marti, G. Metcalfe. A Hennessy-Milner Property for Many-Valued Modal Logics. Proc. of Advances in Modal Logic 2014, pp. 407-420.

[15] A. M. Mironov. Fuzzy Modal Logics. Journal of Mathematical Sciences, Volume 128, Issue 6, pp 3461-3483, 2005.

[16] G. Priest. Many-valued modal logics: a simple approach. Review of Symbolic Logic 1: 190-203, 2008.

[17] A. Vidal, F. Esteva, L. Godo, On modal extensions of product fuzzy logic. Journal of Logic and Computation 27(1): 299-336, 2017. 